\title{
Effets des acides gras conjugués sur les composantes de la dépense énergétique chez la souris et le hamster
}

Patrick EVEN ${ }^{1}$

Jean-Christophe BOUTHEGOURD ${ }^{1}$

Jean-Charles MARTIN²

Annie QUIGNARD BOULANGÉ ${ }^{3}$

${ }^{1}$ UMR INRA/INA P-G 914, Physiologie de la nutrition et du comportement alimentaire,

16, rue Claude Bernard, 75231 Paris Cedex 05

${ }^{2}$ Laboratoire de physiologie de la nutrition, Université Paris-Sud, Bat. 447, campus d'Orsay, 91405 Orsay Cedex

${ }^{3}$ Inserm U465, Institut Biomédical des

Cordeliers, 15, rue de l'Ecole de Médecine, 75270 Paris Cedex 06

Les acides gras conjugués sont des isomères de I'acide linoléique. Parmi eux, l'isomère $9 c, 11 t$, I'acide ruménique est produit de façon naturelle en quantités significatives dans le rumen des bovins puis transféré dans le lait et les tissus, dont il constitue la source nutritionnelle principale pour I'homme [1]. Les CLA ont bénéficié de beaucoup d'attention des dernières années du fait de leurs activités biologiques variées, anticancéreuses, anti-athérosclérotiques et de leur capacité à moduler les fonctions immunitaires $[2,3]$. Dans le cadre qui nous intéresse plus particulièrement, $c^{\prime}$ est surtout le potentiel des CLA à réduire la masse graisseuse et éventuellement à augmenter la masse maigre chez l'animal en croissance, l'adulte obèse et le sujet sous entraînement physique qui a motivé nos études.

Plusieurs mécanismes, non exclusifs, ont été proposés pour essayer d'expliquer les effets des CLA sur la composition corporelle : une augmentation de l'apoptose des cellules adipeuses [4], une diminution de la taille des adipocytes [5], une diminution de la capture d'acides gras par les adipocytes [6], une prise alimentaire réduite, une dépense énergétique augmentée [7-10].

L'une des caractéristiques des effets des CLA qui rend difficile la compréhension de leurs mécanismes d'action, et éventuellement l'extrapolation à l'Homme des résultats expérimentaux sur I'animal, est une grande variabilité des réponses selon l'espèce (rat, souris, hamster, porc, etc.) et les mélanges et quantités de CLA utilisés selon les expériences. Par exemple, il n'a pas été rapporté d'effet notable sur la

\begin{abstract}
We measured the effects of feeding conjugated linoleic acids (CLA) on the various components of energy expenditure in two species known to react differently to CLA treatments, the hamster and the mouse. We measured in particular resting energy expenditure, and the metabolic responses to feeding and treadmill exercise. Our results confirmed the poor responsiveness of hamsters and the high sensibility of mice. In agreement with the lack of effects of CLAs on body weight and body composition in hamsters, we did not find any significant effects on the various components of energy expenditure. Mice responded to CLA feeding by a complete inhibition in the development of the white adipose tissue, but we observed only marginal effects of the components of energy expenditure, and it was difficult to separate the metabolic alterations that resulted from the energy deficit and those that could be specifically attributed to CLAs feeding. However, the possibility that a high spontaneous activity together with the progressive development of a form of insulin resistance participate in the lipoatrophy observed in mice.
\end{abstract}

Key words: conjugated linoleic acids, energy expenditure, hamster, mouse

dépense énergétique de repos chez le rat [5], la truie [11] ou la femme adulte [12], alors qu'au contraire, il semble que les CLA induisent une augmentation significative de ce paramètre chez la souris $[7,9,13]$. Ces données semblent aller de pair avec la simple observation que les CLA ont des effets fulgurants sur la masse grasse des souris alors que leurs effets dans les autres espèces sont nettement plus subtils, si ce n'est carrément absents.

Dans la suite des travaux que nous avons réalisés, nous avons essayé d'aller assez profondément dans la mesure des diverses composantes de la dépense énergétique dans deux espèces animales à la réactivité différentes vis-à-vis des CLA, le hamster et la souris, afin d'essayer de comprendre si l'origine des variabilités interespèces repose sur une action spécifique des CLA sur l'une des composantes énergétiques souvent pas, ou mal, prise en compte dans les études de calorimétrie animale, à savoir, outre la dépense énergétique de totale classiquement étudiée, la dépense énergétique de repos, le niveau d'activité motrice spontanée et éventuellement son coût, et la réponse à l'exercice et aux variations de l'apport alimentaire. Pour ce faire, nous avons utilisé un système de calorimétrie développé spécifiquement pour mesurer ces différentes composantes chez le rat et la souris, qui a la particularité de pouvoir mesurer, puis traiter la mesure simultanée des échanges respiratoires, de l'activité motrice et de la prise alimentaire afin de décomposer la dépense énergétique totale dans ses diverses sous-composantes $[14,15]$.

\section{Méthodologies générales utilisées au cours de ces études}

Les mesures de dépense énergétique ont été réalisées sur un système de mesure des échanges respiratoires bien décrit dans plusieurs publications et dont la pièce principale est une chambre métabolique incluant des capteurs de mesures pour la prise alimentaire et l'activité motrice. Pour la mesure de la réponse à l'exercice, cette chambre peut être temporairement remplacée par un tapis roulant [16]. Le protocole de base utilisé pour déterminer les diverses composantes de la dépense énergétique chez la souris et le hamster consistait à loger les animaux dans la chambre métabolique en début de nuit, sans nourriture, de façon à ce que le lendemain matin les animaux soient à jeun, ce qui donnait la mesure du métabolisme de base. Les animaux étaient ensuite soumis à un exercice d'une heure après transfert dans le tapis roulant (10 h à $12 \mathrm{~h}$ ), ce qui donnait la mesure des réponses métaboliques à l'exercice, puis laissé deux heures en récupération avant de recevoir un repas de taille standardisée, ce qui donnait accès à la mesure de la thermogenèse post-prandiale. Le traitement des données par une suite de programmes informatiques permettait aussi d'extraire en continu, tout au long de l'expérience, l'intensité de l'activité motrice et les variations de la dépense énergétique directement associées à ce comportement. Ce protocole type permettait donc d'extraire de chaque étude le métabolisme basal, le métabolisme associé à l'exercice, la 
thermogenèse post-prandiale, l'intensité et le coût énergétique de l'activité spontanée. Pour chacune de ces composantes, la part relative des taux d'oxydation glucidiques et lipidiques était déterminée à l'aide d'équations stœchiométriques classiques de calorimétrie. Les hamsters ont été étudiés par groupe de deux dans le modèle de chambre calorimétrique pour rat, les souris ont été étudiées par groupe de trois dans le modèle de chambre métabolique pour souris.

\section{Expérience 1 : effets des CLA chez le hamster}

\section{Animaux et procédure}

L'étude a été réalisée chez des hamsters mâles, élevés dans l'animalerie du Laboratoire de physiologie de la nutrition, Université Paris Sud, Orsay, dans des conditions contrôlées d'environnement : température $22^{\circ} \mathrm{C} \pm 1$, humidité $70 \%$, et éclairage selon un cycle artificiel $12 \mathrm{~h}$ : $12 \mathrm{~h}$, nuit de $19 \mathrm{~h} 30$ à $7 \mathrm{~h} 30$ le lendemain. Les animaux étaient maintenus sur un régime standard non purifié (UAR AO4, Épinay-surOrge, France) jusqu'à l'âge de 7 semaines, puis soumis à un régime purifié contenant (en énergie) $48,9 \%$ de glucides, $17,9 \%$ de protéines (caséine) et $32,9 \%$ de lipides pendant deux semaines avant le début des mesures. Les hamsters, jusque-là logés en cages collectives (10 hamsters par cage) furent ensuite logés en cages individuelles et répartis en trois groupes recevant : 1 ) le régime témoin (Groupe $T), 2$ ) le régime témoin additionné de l'isomère $9 c, 11 T$ ( $1,6 \%$ en énergie) (groupe $R$ pour ruménique), 3) le régime témoin additionné d'un mélange de CLA $(47,2 \% 9 \mathrm{c} 11 \mathrm{t}, 48,2 \%$ $10 \mathrm{t} 12 \mathrm{c}$ et $4,6 \%$ d'autres isomères) (groupe $M$ pour Mélange). Les hamsters furent maintenus sur ce régime pendant 6-8 semaines avant le début des mesures de dépense énergétique. La description et l'analyse détaillées de cette étude ont été publiées en 2002 [17]

\section{Résultats}

L'ensemble des résultats est résumé sur les figures 1 et 2. La mesure de la prise alimentaire et du gain de poids a donné des résultats semblables dans les trois groupes (résultats non présentés). À l'état basal, c'est-à-dire après la nuit de jeûne et avant l'exercice, l'intensité du métabolisme de repos ainsi que les taux d'oxydation des glucides et des lipides étaient comparables dans les trois groupes, ce qui indiquait une absence d'effet du traitement sur le métabolisme de base des hamsters (valeurs du métabolisme avant le repas et avant l'exercice sur les figures 1 et 2). De plus, les réponses des hamsters au repas (figure 1) comme à l'exercice (figure 2) furent très comparables dans les trois

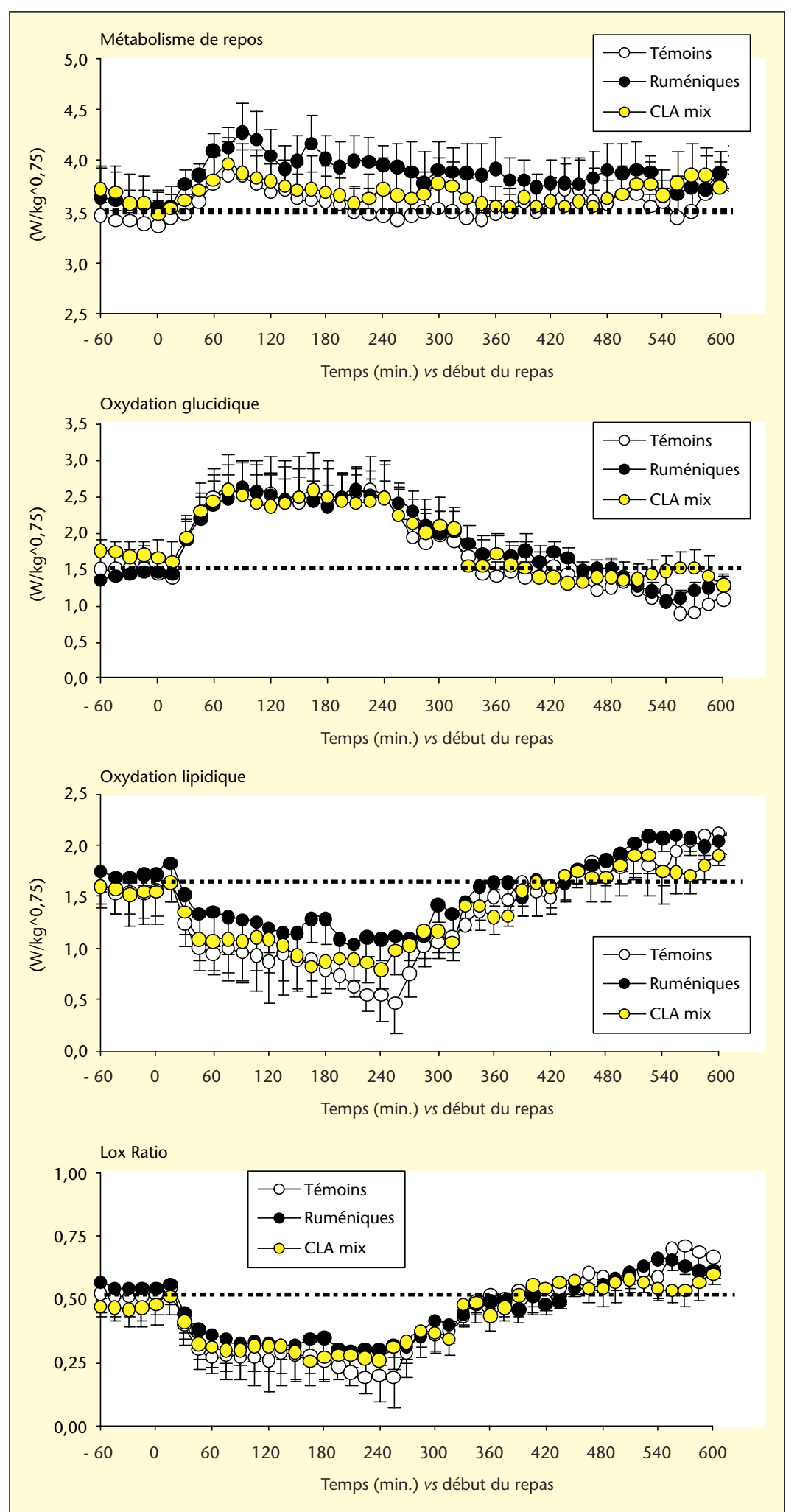

Figure 1. Changements induits par le repas test sur le métabolisme de repos, l'oxydation des glucides (Gox), l'oxydation des lipides (Lox) et la participation relative des lipides au métabolisme (Lox ratio).

Au moment des mesures, les hamsters avaient été nourris pendant 3-4 semaines avec le régime témoin (Témoins), le régime enrichi par l'isomère 9c,11t (Ruméniques) ou par le mélange d'acides gras conjugués (CLA mix). Le repas test est donné au temps 0 et a été ingéré en moins de 15 minutes. Les mesures ont été effectuées sur des hamsters logés par deux. Valeurs = moyennes \pm erreurs moyennes de 5 paires de hamsters. 


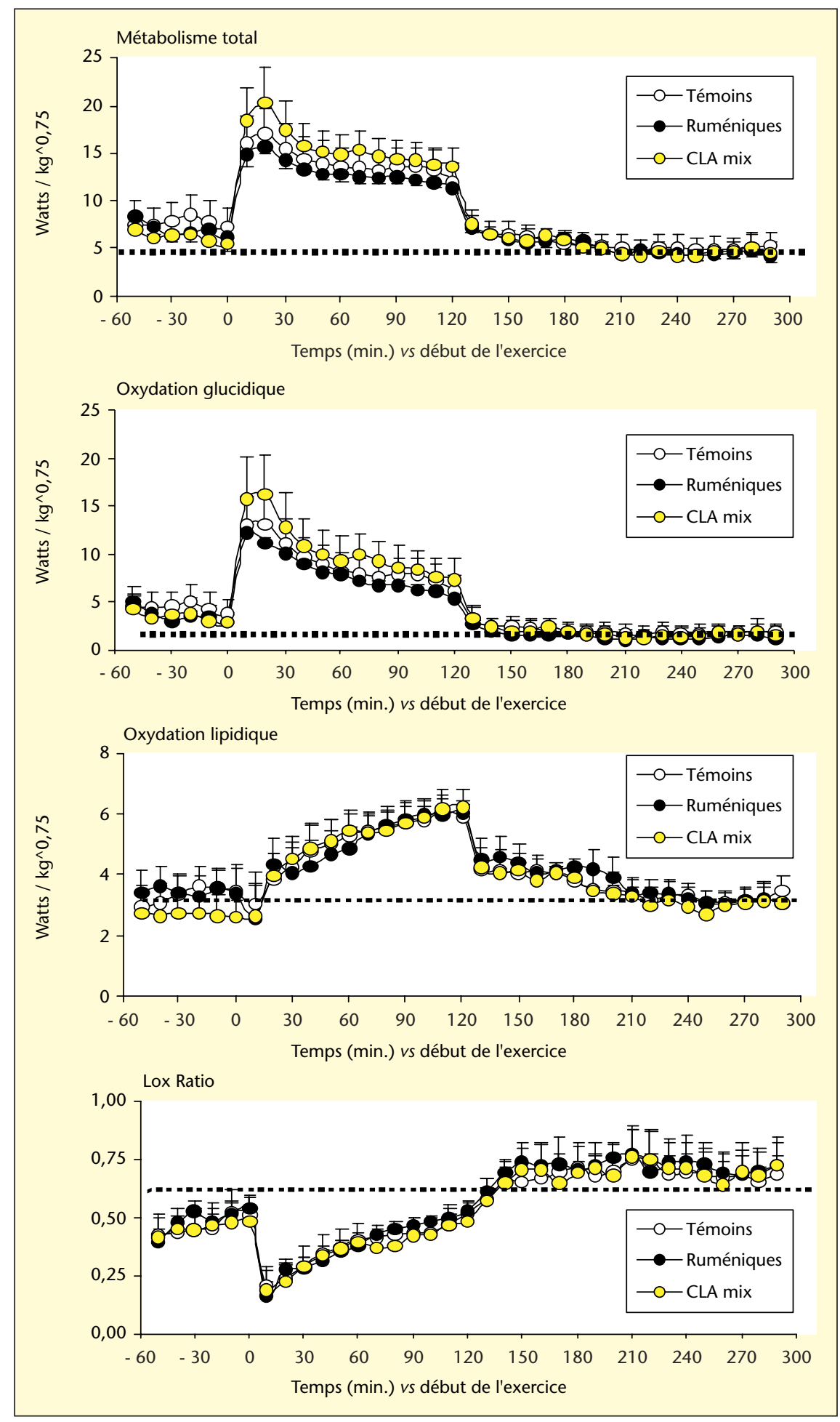

Figure 2. Changement induits en relation avec l'introduction d'un exercice de deux heures sur la dépense énergétique totale (Gox+Lox), I'oxydation des glucides (Gox), I'oxydation des lipides (Lox) et le Lox ratio (voir figure1). Mêmes hamsters que ceux de la figure 1.

groupes confirmant l'absence d'effet du traitement aux CLA sur le métabolisme énergétique. En ce qui concerne l'exercice en particulier, non seulement les réponses à l'exercice étaient identiques, mais la cinétique de retour post- très semblables (figure 2). Pris dans leur ensemble, ces résultats indiquent que les CLA n'affectent aucunement la régulation du métabolisme énergétique chez le hamster. La seule différence que nous avons pu observer entre les différents groupes de hamsters était liée à l'intensité et au coût de l'activité motrice spontanée qui tendait à être légèrement plus faible dans le groupe Ruméniques que dans le groupe Témoins $(P=0,054)$ ou dans le groupe CLA mix $(P=0,15)$ (données non présentées). Cette diminution était plus spécifiquement due à une diminution de l'intensité de l'oxydation des lipides liée à l'activité motrice spontanée dans le groupe Ruméniques, c'est-à-dire paradoxalement, un phénomène qui doit tendre à diminuer l'utilisation des graisses dans ce groupe.

\section{Discussion}

Cette expérience démontre clairement que dans la souche de hamster que nous avons étudiée, les CLA n'affectent que très marginalement les composantes de la dépense énergétique, et pour le groupe ruménique dans un sens qui va plutôt vers une diminution des taux d'oxydation des lipides. Ce résultat va d'ailleurs dans le sens de données acquises parallèlement, indiquant que la taille des dépôts adipeux n'était pas modifiée de façon significative, mais que le contenu corporel total en triglycérides relativement au contenu corporel en eau (mesuré par RMN) était légèrement augmenté dans le groupe R. Bien que certaines études aient montré une baisse du tissu adipeux $[18,19]$, ces résultats confirment la variabilité des réponses selon les souches de hamsters [20-22]. Une analyse détaillée a été faite dans un article publié en 2002 par Bouthegourd et al. [17].

\section{Expérience 2 : effets des CLA chez la souris}

\section{Animaux et procédures}

L'étude a été réalisée chez des souris mâles C57BI/6 (Dépré, France) âgées de 6 semaines au début de l'étude et logées à 3 par cage dans un environnement contrôlé $\left(22 \pm 1^{\circ} \mathrm{C}\right.$, cycle de lumière artificielle de $12 \mathrm{~h}$ de jour et $12 \mathrm{~h}$ de nuit (nuit entre $18: 00$ et $06: 00$ ). Les souris ont été entretenues sur un régime semisynthétique standard de composition suivante (ingrédients en $\mathrm{g} / \mathrm{kg}$ ) : amidon de maïs, 480 ; saccharose, 150 ; caséine "vitamin-free», 210; huile de colza, 50; cellulose, 50; mélange minéral, 35 ; mélange vitaminique, 10. Le traitement aux CLA et au mélange CLAGuarana a été effectué en ajoutant au régime $1 \%$ (en poids) une mixture de CLA (groupe CLA) ou le même mélange de $1 \%$ de CLA 
additionné de Guarana (8\% de caféine) (groupe CG) [23]. Les mélanges de CLA et de $\mathrm{CLA}+$ Guarana nous ont été fournis gracieusement par Loders-Croklaan (Wormerveer - PaysBas) et Physcience/Orsinia (Paris, France). Les souris ont été réparties en six groupes, chacun composé de 5 lots de trois souris. Tous les groupes ont été maintenus sur le régime de base pendant 10 jours, puis deux groupes furent maintenus sur le régime témoin (CT), deux groupes furent complémentés aux CLA (CLA), et les deux derniers groupes furent complémentés avec le mélange CLA-Guarana (CG). Les composantes de la dépense énergétique furent mesurées dans trois des six groupes après 3 jours de traitement (CT, CLA, CG) puis dans les trois derniers groupes après 15 jours de traitement. Nous avons réalisé cette double mesure pour tenir compte du fait que les CLA ont un effet très important sur l'évolution de I'adiposité des souris, et essayer de discriminer ainsi les effets des CLA proprement dits (mesures à trois jours) des effets combinés des CLA et de leurs conséquences à long terme sur la régulation énergétique.

Les souris ont été sacrifiées à leur sortie de la chambre métabolique pour l'analyse de leur composition corporelle. Pour ce faire, les souris furent profondément anesthésiées au pentobarbital (48 mg $/ \mathrm{kg}$ ) (Sanofo Santé), puis le foie, la carcasse, les dépôts adipeux (épididymaire, rétropéritonéal, sous-cutané) et le tissu adipeux brun furent disséqués et pesés au 1/100 de gramme. Le contenu du foie en triglycéride fut aussi mesuré sur un homogénat de foie à I'aide d'un kit enzymatique (Infinity triglyceride reagent, Sigma Diagostics, St-Louis, MO, ÉtatsUnis).

\section{Résultats}

\section{Poids et composition corporelle}

Les poids corporels totaux, des carcasses et des foies sont restés comparables dans les différents groupes après trois jours comme après 15 jours de traitement (tableau 1). Cependant, le contenu hépatique en triglycérides a été réduit par les traitements aux CLA et CG, ceci dès après trois jours de traitement. Les poids des dépôts adipeux blancs et bruns étaient trop faibles pour pouvoir être mesurés avec précision après trois jours de traitement, y compris dans le groupe témoin. Après 15 jours de traitement, les témoins avaient accumulé significativement plus de graisse que les groupes traités aux CLA et au mélange CG. De même, le poids du tissu adipeux brun (TAB) était significativement plus élevé chez les témoins.

Les groupes CLA et CG ayant donné des résultats très semblables sur le plan des réponses métaboliques, leurs données ont été traitées au sein d'un seul groupe (CLA). II y a donc 5
Tableau 1. Composition corporelle mesurée dans les trois groupes de l'expérience (moyenne \pm erreurs moyennes) Les données ont été comparées par analyse de variance au seuil $P<0,05$ suivit si nécessaire par un test post-hoc de Tukey. Les moyennes, sur la même ligne ne partagent pas de lettre commune sont significativement différentes.

\begin{tabular}{|lccc|}
\hline & Témoins & CLA & CG \\
\hline Après 3 jours de traitement & & & \\
Poids (g) & $16.4 \pm 0,4$ & $17,3 \pm 0,3$ & $16,7 \pm 0,3$ \\
Foie (g) & $0.66 \pm 0,02$ & $0,66 \pm 0,02$ & $0,66 \pm 0,03$ \\
TC du foie (mg/g) & $21.3 \pm 0,6(\mathrm{a})$ & $8,1 \pm 2,6(\mathrm{~b})$ & $7,1 \pm 4,3(\mathrm{~b})$ \\
Carcasse (g) & $8.7 \pm 0,2(\mathrm{a})$ & $9,5 \pm 0,2(\mathrm{~b})$ & $9,0 \pm 0,2(\mathrm{ab})$ \\
Carcasse/Pds \% & $53.1 \pm 0,5$ & $54,7 \pm 0,7$ & $53,6 \pm 0,5$ \\
Après 15 jours de traitement & & & \\
Poids (g) & $22,7 \pm 0,8$ & $20,09 \pm 0,8$ & $20.06 \pm 0,6$ \\
Foie (g) & $0,90 \pm 0,05$ & $1,05 \pm 0,05$ & $0.97 \pm 0.04$ \\
TC du foie(mg >/g) & $24,7 \pm 4,9(\mathrm{a})$ & $7,1 \pm 3,1(\mathrm{~b})$ & $5,5 \pm 2,1(\mathrm{~b})$ \\
TA (mg) & $897 \pm 134(\mathrm{a})$ & $151 \pm 18(\mathrm{~b})$ & $198 \pm 31(\mathrm{~b})$ \\
Epididymaire & $245 \pm 29(\mathrm{a})$ & $37 \pm 10(\mathrm{~b})$ & $56 \pm 10(\mathrm{~b})$ \\
Rétroperitoneal & $88 \pm 17(\mathrm{a})$ & $21 \pm 4(\mathrm{~b})$ & $23 \pm 3(\mathrm{~b})$ \\
Sous-cuténa & $564 \pm 98(\mathrm{a})$ & $93 \pm 22(\mathrm{~b})$ & $119 \pm 20(\mathrm{~b})$ \\
TAB (mg) & $69 \pm 9(\mathrm{a})$ & $15 \pm 9(\mathrm{~b})$ & $12 \pm 7(\mathrm{~b})$ \\
Carcasse (g) & $11,1 \pm 0,3$ & $10,9 \pm 0,4$ & $10,4 \pm 0,4$ \\
Carcasse/ TA & $14,6 \pm 2,2(\mathrm{a})$ & $78,5 \pm 10,0(\mathrm{~b})$ & $64,7 \pm 12,0(\mathrm{~b})$ \\
Carcasse/ Pds \% & $49,0 \pm 0,2$ & $52,2 \pm 0,4$ & $50,5 \pm 0,9$ \\
\hline
\end{tabular}

Poids $=$ poids corporel, $T A=$ tissu adipeux blanc (épididymaire + rétropéritonéal + sous-cutané), $T A B=$ tissu adipeux brun. individus pour le groupe témoin et 10 individus pour le groupe CLA.

\section{Composantes de la dépense énergétique}

La figure 3 donne un exemple d'enregistrement individuel à partir desquels ont été déterminés les résultats de l'étude sur les composantes de la dépense énergétique. Sur cette figure, nous avons localisé les différents moments clés de l'expérience au cours desquels nous avons déterminé les mesures de la dépense énergétique et les vitesses d'oxydation des glucides

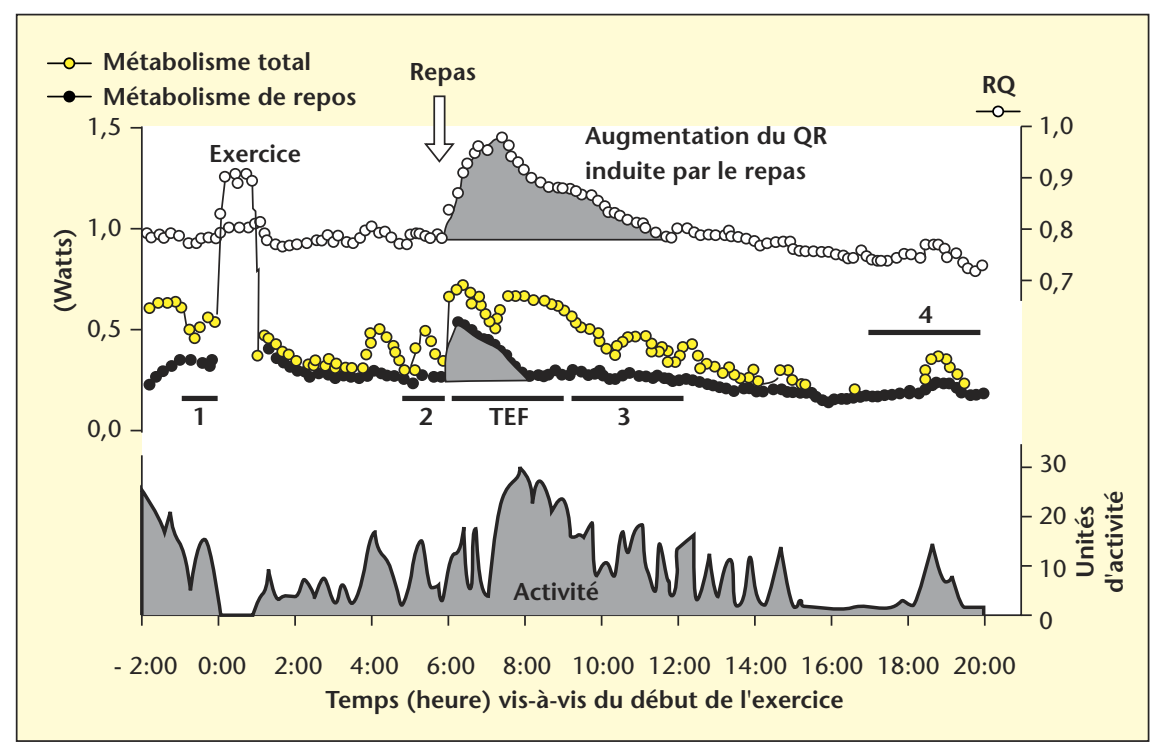

Figure 3. Exemple d'un enregistrement individuel résultant d'une journée de mesure en chambre métabolique (données retravaillées à 15 minutes d'intervalle à partir des mesures originales faites à 10 secondes d'intervalle). $Q R$ : quotient respiratoire, TEF : Effet thermogénique du repas = augmentation du métabolisme de repos induite par I'ingestion du repas. L'espace qui sépare les courbes de métabolisme de repas et de métabolisme total rend compte de la dépense énergétique liée à l'activité motrice spontanée. Sur cette figure sont repérées les 4 périodes de l'expérience que nous avons analysées plus spécifiquement : 1)- la période pré-exercice, 2)-la période pré-repas, TEFla thermogenèse post-prandiale, 4- la période de jeûne. 
Tableau 2. Métabolisme de repos (moyennes \pm erreur moyenne) mesuré pendant les quatre périodes-clés de l'étude en chambre de calorimétrie (voir la figure 1 pour les détails) Les données ont été comparées avec le test du $t$ de Sudent.

\begin{tabular}{|c|c|c|c|}
\hline Watts & Témoins $(n=5)$ & $\operatorname{CLA}(n=10)$ & $P$ \\
\hline \multicolumn{4}{|c|}{ Après 3 jours de traitement } \\
\hline \multicolumn{4}{|c|}{ Métabolisme de repos } \\
\hline Pré-Exercice & $0,30 \pm 0,03$ & $0,32 \pm 0,05$ & $=0,734$ \\
\hline Post-Exercice & $0,25 \pm 0,02$ & $0,26 \pm 0,02$ & $=0,824$ \\
\hline Post-TEF & $0,28 \pm 0,02$ & $0,26 \pm 0,01$ & $=0,458$ \\
\hline Jeûne & $0,18 \pm 0,03$ & $0,12 \pm 0,02$ & $<0,070$ \\
\hline \multicolumn{4}{|c|}{ Oxydation glucidique } \\
\hline Pré-Exercice & $-0,02 \pm 0,02$ & $-0,01 \pm 0,02$ & $=0,815$ \\
\hline Post-Exercice & $0,07 \pm 0,03$ & $0,06 \pm 0,01$ & $=0,765$ \\
\hline Post-TEF & $-0,04 \pm 0,03$ & $0,05 \pm 0,03$ & $<0,068$ \\
\hline Jeûne & $0.03 \pm 0,01$ & $0,05 \pm 0,01$ & $=0,197$ \\
\hline \multicolumn{4}{|c|}{ Oxydation lipidique } \\
\hline Pré-Exercice & $0,32 \pm 0,03$ & $0,33 \pm 0,05$ & $=0,797$ \\
\hline Post-Exercice & $0,19 \pm 0,04$ & $0,20 \pm 0,02$ & $=0,796$ \\
\hline Post-TEF & $0,33 \pm 0,05$ & $0,22 \pm 0,03$ & $<0,053$ \\
\hline Jeûne & $0,15 \pm 0,03$ & $0,07 \pm 0,02$ & $<0,060$ \\
\hline \multicolumn{4}{|c|}{ Après 15 jours de traitement } \\
\hline \multicolumn{4}{|c|}{ Métabolisme } \\
\hline Pré-Exercice & $0,50 \pm 0,01$ & $0,45 \pm 0,03$ & $=0,361$ \\
\hline Post-Exercice & $0,41 \pm 0,02$ & $0,30 \pm 0,02$ & $<0,009$ \\
\hline Post-TEF & $0,43 \pm 0,05$ & $0,29 \pm 0,01$ & $<0,003$ \\
\hline Fast & $0,34 \pm 0,04$ & $0,10 \pm 0,01$ & $<0,000$ \\
\hline \multicolumn{4}{|c|}{ Oxydation glucidique } \\
\hline Pré-Exercice & $0,06 \pm 0,04$ & $0,05 \pm 0,01$ & $=0,910$ \\
\hline Post-Exercice & $0,16 \pm 0,05$ & $0,04 \pm 0,02$ & $<0,020$ \\
\hline Post-TEF & $0.02 \pm 0,04$ & $0,09 \pm 0,02$ & $=0,129$ \\
\hline Fast & $0.07 \pm 0,04$ & $0,03 \pm 0,01$ & $=0,299$ \\
\hline \multicolumn{4}{|c|}{ Oxydation lipidique } \\
\hline Pré-Exercice & $0,44 \pm 0,05$ & $0,40 \pm 0,03$ & $=0,477$ \\
\hline Post-Exercice & $0,24 \pm 0,06$ & $0,26 \pm 0,04$ & $=0,841$ \\
\hline Post-TEF & $0,41 \pm 0,05$ & $0,21 \pm 0,02$ & $<0,001$ \\
\hline Fast & $0,28 \pm 0,01$ & $0,07 \pm 0,01$ & $<0,000$ \\
\hline
\end{tabular}

$\mathrm{TEF}=$ Effet thermique du repas.

(Gox) et des lipides (Lox) qui sous-tendaient la dépense énergétique.

L'intensité du métabolisme à l'état basal a été mesurée à trois moments différents de la période d'enregistrement en chambre métabolique (figure 3 ), en fin de nuit, soit après environ 12 heures de jeûne, immédiatement avant le repas test soit après environ 15 heures de jeûne aggravés d'une heure d'exercice, puis après la disparition de la thermogenèse alimentaire induite par le repas test. Le résultat des mesures est regroupé dans le tableau 2. Ces résultats mettent en évidence que les valeurs estimées du métabolisme de base sont comparables entre les souris témoins et les souris CLA après trois jours de traitement, mais que ces valeurs sont significativement abaissées chez les souris CLA après 15 jours de traitement.

Nous avons aussi mesuré l'intensité du métabolisme de jeûne en mesurant les valeurs du métabolisme de repos en fin d'enregistrement, soit qu'elles consomment en 24 heures). Ces mesures indiquent que dès après trois jours de traitement les souris CLA tendent à réduire leur métabolisme de jeûne, phénomène qui s'accentue très fortement après 15 jours de traitement. Le calcul des taux d'oxydation glucidiques et lipidiques montre que la baisse importante du métabolisme de jeûne mesurée après 15 jours de traitement résulte exclusivement d'une baisse des taux d'oxydation lipidique (tableau 2).

En fait, toutes les souris CLA traitées pendant 15 jours ont été retrouvées en état de torpeur à la fin des mesures en chambre métabolique (inactivité totale, forte baisse de la température corporelle, raideur corporelle, absence de réaction comportementale à la manipulation). Toutes les souris ont cependant retrouvé un comportement normal après avoir été remises dans leur cage habituelle. L'analyse du phénomène de torpeur a été publiée dans un article spécifique [24].

L'amplitude de la thermogenèse post-prandiale (TPP) (tableau 3) mesurée après trois jours de traitement était identique dans les groupes CT et CLA. L'apparition d'une valeur négative dans le calcul de Lox indique une lipogenèse de novo dans les deux groupes en période postprandiale, plus marquée dans le groupe CLA que dans le groupe témoin. Après 15 jours de traitement, la TPP est restée comparable dans les deux groupes, mais d'amplitude plus élevée que celle mesurée après trois jours de traitement et accompagnée de changements dans les variations des taux d'oxydation des glucides et des lipides de plus faible amplitude que ceux mesurés à trois jours. De plus, les amplitudes de variation de Gox et Lox étaient, contrairement aux observations à trois jours, de plus faibles amplitudes dans le groupe CLA que dans le groupe CT.

En ce qui concerne les variations de la dépense énergétique en relation avec l'exercice sur tapis roulant (tableau 4), nous n'avons observé eu un apport énergétique limité au repas test (1 $\mathrm{g}$ de leur régime, soit environ $25 \%$ de ce

Tableau 3. Variations (moyennes \pm erreurs moyennes) induites par le repas test sur le métabolisme de repos, et les oxydations glucidiques et lipidiques. Les données ont été comparées avec le test du t de Student.

\begin{tabular}{|lccc|}
\hline kJ & Témoins $(n=5)$ & CLA $(n=9-10)$ & $P$ \\
\hline Après 3 jours de traitement & & & \\
Thermogenèse post-prandiale (TPP) & $1,39 \pm 0,17$ & $1,41 \pm 0,16$ & $=0,944$ \\
Variations induites par le repas sur & $1,57 \pm 0,47$ & $2,22 \pm 0,37$ & $=0,321$ \\
l'oxydation glucidique & & & $=0,288$ \\
Variations induites par le repas sur & $-0,14 \pm 0,40$ & $-0,71 \pm 0,30$ & \\
l'oxydation lipidique & & & $=0,765$ \\
Après 15 jours de traitement & & $2,01 \pm 0,25$ & $<0,001$ \\
Thermogenèse post-prandiale (TPP) & $1,89 \pm 0,29$ & $0,33 \pm 0,02$ & $<0,072$ \\
Variations induites par le repas sur & $0,52 \pm 0,05$ & $0.16 \pm 0.02$ & \\
I'oxydation glucidique & & & \\
Variations induites par le repas sur & $0,06 \pm 0,05$ & & \\
I'oxydation lipidique & & & \\
\hline
\end{tabular}


aucune différence entre les deux groupes après 3 jours de traitement, mais après 15 jours, les souris CLA ont éprouvé une certaine difficulté à réaliser l'exercice, apparemment du fait d'une difficulté à mobiliser autant de lipides que les souris témoins. La mesure de l'activité spontanée au cours des différentes périodes (tableau 5) a mis en évidence que les souris CLA tendaient à être hyperactives avant et après l'exercice après trois jours de traitement, une tendance qui se maintenait après l'exercice même après 15 jours de traitement alors que les souris tendaient par ailleurs à être moins actives, puis à devenir totalement inactives en fin de période d'enregistrement.

\section{Discussion} souris confirment la forte sensibilité de cette espèce au traitement par les CLA [4]. La mesure des différentes composantes de la dépense
Les mesures que nous avons effectuées chez la

énergétique faite après trois jours de traitement semble cependant $n$ 'indiquer aucun effet particulier des CLA sur les composantes de la dépense énergétique, ce qui pourrait suggérer que la baisse de l'adiposité induite par le traitement aux CLA n'est pas due à une augmentation primaire du métabolisme de repos, et/ou une augmentation de l'oxydation des graisses. II faut cependant rester prudent dans ce type d'interprétation car des changements extrêmement subtils dans l'équilibre entre l'apport et la dépense énergétique, de même que dans les taux d'utilisation des lipides, difficiles à mettre en évidence par des mesures de calorimétrie à court terme, peuvent conduire sur le long terme à des différences importantes de la composition corporelle. Pour essayer de corriger en partie ce manque de sensibilité de la calorimétrie indirecte à révéler des différences subtiles de la dépense énergétique globale de 24 heures, nous avons organisé le protocole en cage

Tableau 4. Augmentations (moyennes \pm erreurs moyennes) du métabolisme total et des taux d'oxydation glucidiques et lipidiques induites pas l'exercice sur tapis roulant. Les données ont été comparées avec le test du t de Student.

\begin{tabular}{|lccc|}
\hline Watts & Témoins $(n=5)$ & CLA $(n=9-10)$ & $P$ \\
\hline $\begin{array}{l}\text { Après 3 jours de traitement } \\
\text { Augmentation du métabolisme en } \\
\text { réponse à l'exercice }\end{array}$ & $0,97 \pm 0,03$ & $1,01 \pm 0,04$ & $=0,50$ \\
$\begin{array}{l}\text { Augmentation de l'oxydation } \\
\text { glucidique en réponse à l'exercice } \\
\text { Augmentation de l'oxydation } \\
\text { lipidique en réponse à l'exercice }\end{array}$ & $0,50 \pm 0,05$ & $0,58 \pm 0,03$ & $=0,13$ \\
$\begin{array}{l}\text { Après 15 jours de traitement } \\
\text { Augmentation du métabolisme en } \\
\text { réponse à l'exercice }\end{array}$ & $0,43 \pm 0,04$ & $0,38 \pm 0,03$ & $=0,42$ \\
$\begin{array}{l}\text { Augmentation de l'oxydation } \\
\text { glucidique en réponse à l'exercice }\end{array}$ & $1,01 \pm 0,05$ & $0,80 \pm 0,09$ & $=0,16$ \\
$\begin{array}{l}\text { Augmentation de l'oxydation } \\
\text { lipidique en réponse à l'exercice }\end{array}$ & $0,47 \pm 0,04$ & $0,49 \pm 0,06$ & $=0,88$ \\
\hline
\end{tabular}

Tableau 5. Mesure de l'énergie dépensée en relation avec l'activité motrice spontanée (moyennes \pm erreurs moyennes) au cours des périodes clefs définies telles qu'à la figure 3. Les données ont été comparées avec le test du $t$ de Student.

\begin{tabular}{|c|c|c|c|}
\hline kJ & Témoins $(n=5)$ & $\operatorname{CLA}(n=9)$ & $P$ \\
\hline \multicolumn{4}{|c|}{ Après 3 jours de traitement } \\
\hline Pre-Exercise & $1,97 \pm 0,20$ & $2,55 \pm 0,18$ & $<0,06$ \\
\hline Post-Exercise & $1,78 \pm 0,22$ & $3,50 \pm 0,60$ & $<0,06$ \\
\hline During TEF & $5,20 \pm 0,64$ & $5,17 \pm 0,21$ & $=0,96$ \\
\hline Post-TEF & $2,04 \pm 0,65$ & $1,46 \pm 0,24$ & $=0,33$ \\
\hline During Fast & $0,80 \pm 0,14$ & $0,57 \pm 0,27$ & $=0,54$ \\
\hline Total & $11,79 \pm 1,12$ & $13,20 \pm 0,77$ & $=0,31$ \\
\hline \multicolumn{4}{|c|}{ Après 15 jours de traitement } \\
\hline Pre-Exercise & $2,22 \pm 0,29$ & $1,52 \pm 0,07$ & $<0,01$ \\
\hline Post-Exercise & $1,19 \pm 0,44$ & $3,20 \pm 0,38$ & $>0,01$ \\
\hline During TEF & $5,76 \pm 0,18$ & $5,02 \pm 0,25$ & $<0,07$ \\
\hline Post-TEF & $4,74 \pm 0,43$ & $1,87 \pm 0,32$ & $<0,00$ \\
\hline During Fast & $1,04 \pm 0,18$ & $0,09 \pm 0,05$ & $<0,00$ \\
\hline Total & $14,95 \pm 0,43$ & $11,68 \pm 0,34$ & $<0,00$ \\
\hline
\end{tabular}

de métabolisme de façon à pouvoir accéder directement à la mesure des différentes composantes de la dépense énergétique. Si l'on se limite à l'analyse des données obtenues après trois jours de traitement, c'est-à-dire avant que des adaptations possibles à un déficit énergétique ne soient susceptibles d'interférer avec les effets propres des CLA, on peut considérer que la dépense énergétique de base n'est pas modifiée par le traitement aux CLA, mais que par contre des altérations peuvent avoir lieu au niveau des composantes de la dépense énergétique en relation avec l'activité physique et la réponse métabolique à l'ingestion d'un repas. En effet, il semble que, au moins pendant les premiers jours de traitement, un certain degré d'hyperactivité peut apparaître chez les souris traitées et induire, si cette hyperactivité n'est pas compensée par une augmentation proportionnelle de la prise alimentaire, un déficit énergétique qui pourrait en partie expliquer l'absence d'accumulation de graisse chez les souris traitées. Les mesures sur tapis roulant semblent par contre indiquer qu'il n'y a pas d'altération propre aux CLA de la réponse métabolique associée au travail musculaire.

En ce qui concerne la réponse métabolique à l'ingestion d'aliments, nous n'avons pas observé d'effet quantitatif sur la dépense énergétique, c'est-à-dire que l'intensité de la thermogenèse alimentaire était la même dans les deux groupes. Cependant, les variations des taux d'oxydation des glucides et des lipides qui sous-tendaient cette thermogenèse semblent être plus intenses chez les souris traitées après seulement trois jours de traitement, une observation qui est contraire à ce que I'on pouvait attendre du fait de l'effet à long terme des CLA. En effet, la forte inhibition des taux d'oxydation post-prandiale des lipides devrait normalement se traduire sur le long terme par un stockage augmenté des lipides. En fait, la réponse à cette incompatibilité de principe est peut-être apportée par les mesures faites à 15 jours qui révèlent un taux d'inhibition post-prandiale de I'oxydation des lipides nettement diminué chez les souris traitées aux CLA, et est donc, là, en parfait accord avec la réduction du stockage des lipides chez les souris traitées. La question à laquelle on ne peut répondre cependant au stade actuel des travaux est de savoir si cet effet observé à 15 jours est le résultat d'un effet propre à long terme des CLA, par exemple la conséquence d'une augmentation de l'insulinorésistance des souris comme il a été évoqué dans certains travaux précédents $[9,13]$, ou tout simplement une conséquence liée aux perturbations métaboliques qui découlent de l'amaigrissement prononcé des souris à ce stade du traitement. En effet, le déficit énergétique induit par 15 jours de traitement aux CLA, semble induire certains effets spécifiques 
tels qu'une diminution accélérée du métabolisme basal dès que la souris subit quelques heures de jeûne, et une tendance prononcée des souris, somme toute logique au vu de l'état des réserves lipidiques, à avoir des difficultés à mobiliser leurs lipides en cas de besoin augmenté tel que l'exercice ou le jeûne. La réduction importante de l'utilisation des lipides en cas de jeûne prolongé a d'ailleurs provoqué un stade de torpeur prolongé chez les souris d'autant plus significatif qu'il faut en principe des stress métaboliques intenses tels que des baisses de la température ambiante ou le blocage simultané de l'oxydation des glucides et des lipides par des animétabolites pour induire un phénomène de torpeur chez la souris [25-27]. Le contraste assez fort observé entre la possibilité, certes un peu réduite mais quantitativement très significative, des souris à mobiliser des lipides au cours de l'exercice, et le blocage quasi total de cette mobilisation quelques heures plus tard, soulève d'ailleurs la question de savoir si nous avons affaire à un phénomène purement passif résultant de la déplétion totale des réserves lipidiques ou, au contraire, à une adaptation active mise en place pour économiser les réserves lipidiques. Dans cette dernière hypothèse, il est envisageable qu'une molécule antilipolytique soit libérée par le tissu adipeux et la caractérisation de cette molécule, si elle existe, pourrait permettre un progrès décisif dans la compréhension des mécanismes de régulation de l'équilibre énergétique.

En conclusion, la mesure des composantes de la dépense énergétique chez la souris traitée au CLA a mis en évidence des effets plus marqués après 15 jours de traitement qu'après seulement trois jours, et il est difficile de dire aujourd'hui si les effets mesurés après 15 jours résultent d'un effet direct des CLA ou d'adaptations à la diminution importante des réserves lipidiques.

Le dernier point qui mérite d'être discuté est l'absence de stéatose hépatique chez les souris de cette étude, une observation qui va à I'encontre des observations précédentes [4]. La raison de cette différence qui nous semble la plus probable est le fait que nous ayons effectué les prélèvements de tissus sur les souris à la fin des mesures de dépense énergétique, donc sur des souris qui avaient subi une période de jeûne prolongée. Un calcul rapide basé sur la dépense énergétique des souris permet d'estimer en effet que la procédure en calorimètre a imposé aux souris la mobilisation d'environ $850 \mathrm{mg}$ de lipides, qui, s'ils ont été mobilisés à partir des réserves hépatiques plutôt que du tissu adipeux, peuvent expliquer l'absence de graisses hépatiques chez les souris traitées de cette étude.

\section{Perspectives}

Les résultats de cette étude confirment bien que les CLA ont des effets sur l'adiposité qui peuvent être très différents selon les espèces. En pratique, il faut convenir que mise à part la souris, les CLA semblent finalement relativement peu efficaces pour favoriser la réduction des dépôts adipeux puisque ni le hamster, ni le rat [5] ni le lapin [28], et pour ce qui nous intéresse en fin de compte, ni l'Homme, ne répondent à l'ingestion de CLA par une réduction significative des dépôts adipeux. En effet, les résultats rapportés pour I'Homme sont soit négatifs, soit plutôt subtils, les auteurs rapportant davantage des «tendances» que des effets vraiment significatifs [29, 30, 12]. La situation est rendue d'autant plus complexe qu'il semble que les différents types de CLA puissent avoir des actions différentes. Par exemple chez la souris [31] et sans doute aussi le hamster [19], seul l'isomère t-10, c-12 semble efficace en même temps qu'il semble que, chez la souris, les effets positifs des CLA puissent perdurer après que les CLA aient été retirés de I'alimentation [9]. Plus généralement, il semble que les CLA puissent agir selon des mécanismes différents en fonction des espèces, ce qui semble une hypothèse raisonnable au vu des différences de réponses des différentes espèces. Chez la souris, des études in vitro ont montré une augmentation de la lipolyse et de la bêtaoxydation en réponse aux CLA [32], de même qu'une augmentation de l'apoptose adipocytaire [4], ce qui peut être un mécanisme extrêmement intéressant pour le traitement à long terme de l'obésité et expliquer le maintien d'une adiposité réduite chez les souris même après que l'apport nutritionnel des CLA ait été réduit. Un tel phénomène d'apoptose n'a cependant pas été rapporté dans d'autres espèces. Chez le rat par exemple, les CLA semblent réduire la taille adipocytaire sans réduction du nombre des adipocytes [5], ce qui sous-entend que si l'apport de CLA est arrêté, les adipocytes pourraient reprendre leur taille normale. Un mécanisme de ce type hypothèque l'efficacité potentielle des CLA dans le traitement à long terme de l'obésité, surtout si I'on considère en parallèle leurs effets potentiellement délétères sur l'insulinorésistance. De plus, chez le rat [5] et dans certaines expériences chez la souris [29], des augmentations de la synthèse des acides gras ont été rapportées, ce qui semble peu compatible avec la réduction de I'adiposité. Nous avons nous-mêmes observé et discuté plus haut le fait que, après trois jours de traitement, des augmentations plus importantes du quotient respiratoire en réponse au repas test chez les souris traitées, révélatrices $d$ 'un processus de lipogenèse intense qui semblait en effet peu compatible avec le fait que les CLA réduisaient finalement I'accumulation de graisse.

Enfin, in vivo, une augmentation de la dépense énergétique susceptible de favoriser un bilan énergétique négatif n'a été rapporté que chez la souris [29]. Ces résultats ne sont pas complètement incompatibles avec les résultats que nous présentons ici, dans la mesure où ils ont été obtenus sur la dépense énergétique totale de souris à l'état nourri. L'extrapolation de nos résultats dans des conditions expérimentales identiques (même dépense énergétique basale, mais hyperactivité) va aussi dans le sens d'une augmentation de la dépense énergétique totale chez la souris nourrie.

Enfin, pour revenir et conclure sur les effets potentiellement néfastes des CLA sur I'insulinorésistance, les résultats acquis actuellement suggèrent qu'ils sont particulièrement manifestes chez la souris $[9,13]$, moins chez le rat [33] ou le porc [34], et quasi absents chez l'Homme (Medina, 2000). On peut, en comparant cette échelle à celle de l'efficacité des CLA à réduire I'adiposité chez la souris, se demander si l'efficacité des CLA dans cette espèce ne passe pas justement pas le développement rapide d'une forme d'insulinorésistance sévère, peut-être produite pas la production des TNF $\alpha$, euxmêmes probablement à l'origine de l'apoptose [4]. Les résultats de l'étude présente semblent effectivement indiquer qu'après 15 jours de traitement, les souris CLA présentaient une insulinorésistance sévère révélée par la faiblesse des variations des taux d'oxydation des glucides et des lipides en réponse au repas test. II faut admettre que si l'efficacité des CLA sur la composition corporelle passe par un tel mécanisme, leur utilisation pour lutter contre l'obésité chez l'Homme est peu envisageable.

\section{RÉFÉRENCES}

1. KEPLER CR, HIRONS KP, MACNEILL \|, TOVE $S B$. Intermediates and products of the biohydrogenation of linoleic acid by Butyrivibrio fibrisolvens. J Biol Chem 1966 ; 241 : 1350-4.

2. BANNI S, MARTIN JC. Conjugated linoleic acid and metabolites. In : Christie WW, Sébédio JL, eds. Trans fatty acids in human nutrition. Dundee, Scotland : The Oily Press, 1998 : 262-301.

3. TURINIM, MARTIN JC. Conjugated linoleic acid. In : Gunstone FD, ed. Structured lipids. New York : Marcel Decker, 2001 : 251-84.

4. TSUBOYAMA-KASAOKA N, TAKAHASHIM, TANEMURA K, et al. Conjugated linoleic acid supplementation reduces adipose tissue by apoptosis and develops lipodystrophy in mice. Diabetes 2000 ; 49 : 1534-42.

5. AZAIN MJ, HAUSMAN DB, SISK MB, et al. Dietary conjugated linoleic acid reduces rat adipose tissue cell size rather than cell number. / Nutr $2000 ; 130$ : 1548-54. 
6. PARIZA MW, PARK Y, FUSHIKI T. A Single Oral Administration of Conjugated Linoleic Acid enhanced energy metabolism in mice. Lipids $2001 ; 36: 583-7$.

7. WEST DB, DELANY JP, CAMET PM, BLOHM F, TRUETT AA, SCIMECA J. Effects of conjugated linoleic acid on body fat and energy metabolism in the mouse. Am / Physiol 1998; 275 : R667-R672

8. DELANY JP, WEST DB. Changes in body composition with conjugated linoleic acid. / Am Coll Nutr $2000 ; 19$ : 487S-493S.

9. WEST DB, BLOHM FY, TRUETT AA, DELANY JP. Conjugated linoleic acid persistently increases total energy expenditure in AKR/J mice without increasing uncoupling protein gene expression. J Nutr $2000 ; 130$ : 2471-7.

10. TERPSTRA AH. Differences between human and mice in efficacy of the Body fat lowering effect of conjugated linoleic acid : role of metabolic rate. J Nutr 2001 ; 131 : 2067-8.

11. MÜLLER HL, KIRCHGESSNER FX, ROTH FX, STANGL GI. Effect of conjugated linoleic acid on energy metabolism in growing-finishing pigs. J Anim Physiol Anim Nutr (Berl) $2000 ; 83$ : 85-94.

12. ZAMBELL KL, KEIM NL, VAN LOAN MD, et al. Conjugated linoleic acid supplementation in humans: Effects on body composition and energy expenditure. Lipids 2000 ; 35 : 777-82.

13. DELANY JP, BLOHM F, TRUETT AA, SCIMECA JA, WEST DW. Conjugated linoleic acid rapidly reduces body fat content in mice without affecting energy intake. Am / Physiol 1999; 276 : R1172-R1179.

14. EVEN PC, PERRIER E, AUCOUTURIER JL, NICOLAIDIS S. Utilisation of the method of Kalman filtering for performing the on-line computation of background metabolism in the free-moving, free-feeding rat. Physiol Behav $1991 ; 49: 177-87$

15. EVEN PC, MOKHTARIAN A, PELE A. Practical aspects of indirect calorimetry in laboratory animals. Neurosci Biobehav Rev 1994 ; 18 : 43547.
16. EVEN PC, RIETH N, ROSEAU S, LARUEACHAGIOTIS C. Substrate oxidation during exercise in the rat cannot fully account for training-induced changes in macronutrients selection. Metabolism 1998 ; 47 : 777-82.

17. BOUTHEGOURD JC, EVEN PC, GRIPOIS D, et al. A CLA mixture prevents trihlyceride accumation without affecting energy expenditure in Syriam hamsters. J Nutr 2002 ; 132 : 2682-9.

18. DE DECKERE EA, VAN AMELSVOORT JM, MCNEILL GP, JONES P. Effects of conjugated linoleic acid (CLA) isomers on lipid levels and peroxisome proliferation in the hamster. $\mathrm{Br}$ Nutr $1999 ; 82$ : 309-17.

19. GAVINO VC, GAVINO G, LEBLANC M], TUCHWEBER B. An isomeric mixture of conjugated linoleic acids but not pure cis-9, trans11-octadecadienoic acid affects body weight gain and plasma lipids in hamsters. I Nutr $2000 ; 130: 27-9$

20. DE DECKERE EMA, D.E. Fouw NI, RiskesHoitinga J, Van Nielen WGL, Blonk CG. Effect of an atherogenic diet on lipoprotein cholesterol profile in the F1B hybrid hamster. Atherosclerosis $1993 ; 103: 291-4$.

21. FEREZOU ], COMBETTES-SOUVERAIN M, SOUIDI, et al. Cholesterol, bile acid, and lipoprotein metabolism in two strains of hamster, one resistant, the other sensitive (LPN) to sucrose-induced cholelithiasis. / Lipid Res 2000 ; 41 : 2042-54.

22. SOUIDI M, COMBETTES-SOUVERAIN M, MILLIAT F. Hamsters predisposed to sucrose- induced cholesterol gallstones (LPN strain) are more resistant to excess dietary cholesterol than hamsters that are not sensitive to cholelithiasis induction. J Nutr 2001 ; 131 : 1803-11.

23. GRAHAM TE, SPRIET LL. Performance and metabolic responses to a high caffeine dose during prolonged exercise. / Appl Physiol 1991 ; $71(6): 2292-8$

24. BOUTHEGOURD JC, MARTIN JC, GRIPOIS D, ROSEAU S, TOME D, EVEN PC. Fat-depleted CLA-treated mice enter torpor after a short period of fasting. Appetite 2004 ; 42 : 91-8.
25. GAVRILOVA O, LEON LR, MARCUSSAMUELS B, et al. Torpor in mice is induced by both leptin-dependent and -independent mechanisms. Proc Natl Acad Sci USA 1999 ; 96(25) : 14623-8.

26. STAMPER JL, DARK J. Metabolic fuel availability influences thermoregulation in deer mice (Peromyscus maniculatus). Physiol Behav 1997 ; 61(4) : 521-4.

27. WEBB GP, JAGOT SA, JAKOBSON ME. Fastinginduced torpor in Mus musculus and its implications in the use of murine models for human obesity studies. Comp Biochem Physiol 1982 ; 72(1) : 211-9; (A).

28. LEE KN, KRITCHEVSKY D, PARIZA MW. Conjugated linoleic acid and atherosclerosis in rabbits. Atherosclerosis 1994 ; 108 : 19-25.

29. ATKINSON RL. Conjugated linoleic acid for altering body composition and treating obesity. In: Yurawecz MP, Mossoba MM, Kramer JKG, et al., eds. Advances in Conjugated Linoleic Acid Research. Champaign, IL : AOCS Press, 1999 : 328-53; 1.

30. BLANKSON H, STAKKESTAD JA, FAGERTUN H, et al. Conjugated linoleic acid reduces body fat mass in overweight and obese humans. J Nutr $2000 ; 130$ : 2943-8.

31. PARK Y, STORKSON JM, ALBRIGHT KJ, et al. Evidence that the trans-10, cis-12 isomer of conjugated linoleic acid induces body composition changes in mice. Lipids $1999 ; 34$ : 23541.

32. PARK Y, ALBRIGHT KI, LIU W, et al. Effect of conjugated linoleic acid on body composition in mice. Lipids 1997 ; 32 : 853-8.

33. HOUSEKNECHT KL, VANDEN HEUVEL JP, MOYA-CAMARENA SY, et al. Dietary conjugated linoleic acid normalizes impaired glucose tolerance in the Zucker diabetic fatty fa/fa rat. Biochem Biophys Res Commun 1998 ; 244 : 67882.

34. STANGL Gl, MULLER H, KIRCHGESSNER M. Conjugated linoleic acid effects on circulating hormones, metabolites and lipoproteins, and its proportion in fasting serum and erythrocyte membranes of swine. Eur / Nutr 1999; 38 : 271-7. 